\title{
The impact of false positive COVID-19 results in an area of low prevalence
}

\author{
Authors: Brendan Healy, ${ }^{\mathrm{A}}$ Azizah Khan, ${ }^{\mathrm{B}}$ Huria Metezai, ${ }^{\mathrm{B}}$ Ian Blyth ${ }^{\mathrm{C}}$ and Hibo Asad ${ }^{\mathrm{D}}$
}

False negative results in COVID-19 testing are well recognised and frequently discussed. False positive results, while less common and less frequently discussed, still have several adverse implications, including potential exposure of a non-infected person to the virus in a cohorted area. Although false positive results are proportionally greater in low prevalence settings, the consequences are significant at all times and potentially of greater significance in high-prevalence settings. We evaluated COVID-19 results in one area during a period of low prevalence. The consequences of these results are discussed and implications for these results in both high and low prevalence settings are considered. We also provide recommendations to minimise the risk and impact of false-positive results.

KEYWORDS: COVID-19, false positive results, high prevalence, low prevalence

DOI: $10.7861 /$ clinmed.2020-0839

\section{Background}

The UK's COVID-19 testing programme uses real-time reverse transcription polymerase chain reaction (RT-PCR) tests to detect

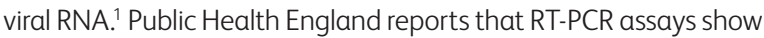
a specificity of over $95 \%$, meaning that up to $5 \%$ of cases are false positives. ${ }^{2}$ The impact of false positive results includes risk of overestimating the COVID-19 incidence, the demand on track and trace, and the extent of asymptomatic infection. ${ }^{3}$ The adverse consequences of false positive results are proportionally greater in low prevalence settings. ${ }^{3}$ We therefore evaluated the clinical implications of false positive results in one area during a time of low prevalence.

\section{Methods}

We collected data from the ICNET platform of patients that tested positive by SARS-CoV-2 RT-PCR including inpatient and

Authors: Aconsultant in microbiology and infectious diseases,

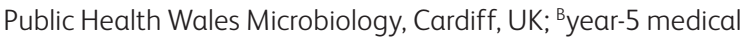
student, Cardiff University, Cardiff, UK; ' Consultant in microbiology and infectious diseases, Public Health Wales, Swansea, UK and Swansea Bay University Health Board, Swansea, UK; Dhealthcare epidemiologist, Public Health Wales, Swansea, UK and Swansea Bay University Health Board, Swansea, UK outpatient testing within the Swansea Bay University Health Board from 20 June 2020 to 21 July 2020 during a period of very low prevalence in this area. Tests were carried out on three platforms: Seegene, Cepheid and Luminex. These platforms use a combination of targets including the $\mathrm{N}$ and $\mathrm{E}$ gene (Cepheid and Luminex) and the N, E and RdRp gene (Seegene). Data were analysed in Excel.

For the purpose of this paper, a result was considered as false positive if it was positive in only a single gene with a $\mathrm{Ct}$ value $>35$. PCR involves repeat cycles of amplification of an RNA target, detected in real time by use of fluorescent dyes. A positive result is generated when the level of fluorescence crosses a predetermined threshold. The positive result can be semi-quantified by the number of cycles required to reach the threshold: the "cycle threshold' (Ct). Low cycle thresholds indicate presence of greater amounts of the target sequence in the sample, and higher viral loads. Ct values vary according to the assay being used but in clinical practice $\mathrm{Ct}<29$ is generally considered a strong positive result, 30-35 a positive result and $>35$ as weak positive result. Further information is also presented to demonstrate the likely false positive results (including repeat swab results where available, absence of symptoms and evolution following the result where available).

\section{Results}

31 throat swabs tested positive for SARS-CoV- 2 by RT-PCR: $n=15$ via Seegene, $n=15$ via Cepheid, and $n=1$ via Luminex.

12 of these patients were symptomatic, while the remaining 19 included five patients screened pre-operatively, five nursing home residents, three on the transplant waiting list, four screened predischarge, one screened pre-delivery and one nursing home key worker.

Our results show that 26/31 were positive at low level $(\mathrm{Ct}>35)$ in a single gene, which is considered as a likely false positive result. However, five were positive in more than one gene: three were positive in two genes (Cepheid $n=2$, Seegene $n=1$ ), and two were positive in three genes (Seegene), which are considered to be likely true positive results (Fig 1).

During the same period, there were 5,079 negative tests; thus a total of 5,110 tests were carried out. The false positive rate (single gene low level positive) in this period was $0.5 \%$ (26/5110), giving a specificity of $99.5 \%(5,079 / 5,105)$. Over the same period, the true positive rate was $16 \%(5 / 31)$. From this data collected in a very low prevalence setting, the negative predictive value was 100\% (no identified false negative results) and the positive predictive 


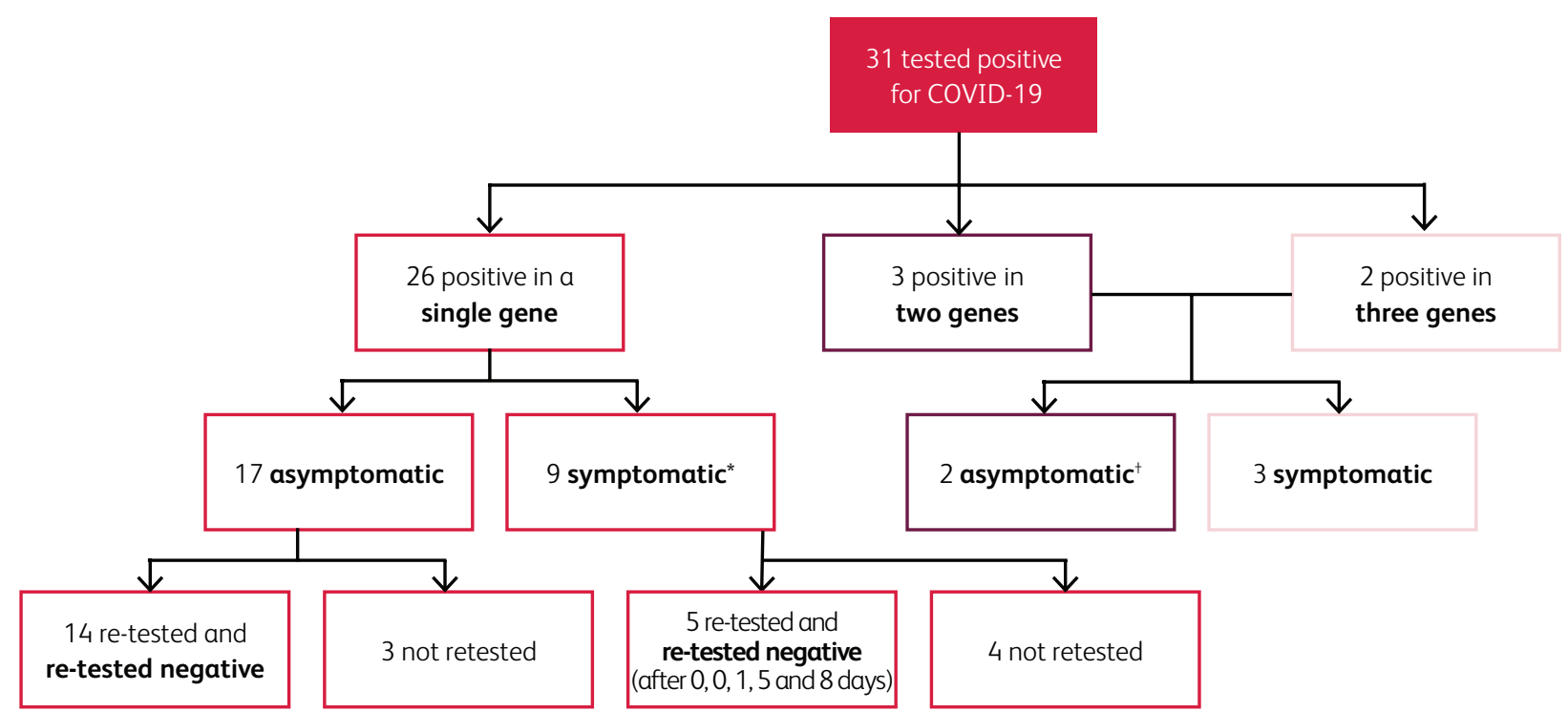

Fig 1. Flow chart summarising the key findings of likely false positive and true positive results. ${ }^{*}$ The range of $\mathrm{Ct}$ values in symptomatic patients 36-43, mean 39.5. One had a previous positive result and the result likely reflected residual genetic material. ${ }^{+}$One was most likely a true positive case. He was asymptomatic at the time of swabbing and tested positive. However, on admission he had CXR changes and lymphopenia. Although he initially tested negative on admission, this was likely a false negative. The other individual was asymptomatic at the time of swabbing and tested positive. Although he was negative on repeat swabs on the same day and two days later, he was positive three months earlier and was antibody positive. We therefore believe the positive result likely reflects the presence of residual RNA.

value was $16 \%(5 / 31)$. It is important to note that as prevalence changes, so does the performance of the test. In particular, the negative predictive value will change dramatically as PCR is said to have a sensitivity in the region of $80 \%$.

\section{Implications of false positive results}

Several potential significant implications for the single-gene low-level false positive results were recognised. Patients on the transplant waiting list were removed from the list for 2 weeks. Some of the patients screened pre-operatively had their surgery delayed. Patients screened pre-discharge were kept in hospital, unnecessarily in many cases. All the low-level, likely false positive results from the nursing home residents and staff generated further activity, including as a minimum re-swabbing but also track and trace of residents and staff in some cases. The results also negatively impacted on staffing levels to varying degrees, affected transfers in and out of the home and caused a distraction from other elements of patient care.

\section{False positive results in low-prevalence settings}

False positive results are proportionally more of an issue when prevalence is low. Prevalence is a measure of how common a disease is in a specified at-risk population at a specific time point or period. ${ }^{5}$ It measures the disease burden for the specified population. ${ }^{5}$ Prevalence affects the pre-test probability of a disease being present and consequently impacts on the positive predictive value (PPV; the probability that subjects with a positive test truly have the disease) and the negative predictive value (NPV; the probability that subjects with a negative test truly do not have the disease). ${ }^{3}$ As the prevalence increases, the PPV increases but the NPV decreases. Similarly, as the prevalence decreases the
PPV decreases while the NPV increases. ${ }^{3}$ The adverse outcomes associated with false positive results will be proportionally greater during periods of low prevalence. ${ }^{3}$ However, the individual impact of false positive results are significant at all times.

\section{False positive results in high-prevalence settings}

Although proportionally false positive results are less of a problem in high-prevalence settings when compared to true positive results, the overall percentage of false positive tests will not change. If testing increases as a consequence of high prevalence, the absolute number of false positive tests will also increase. A false positive result is less likely to be detected during times of high prevalence as the result will receive less scrutiny.

False positive results in this setting can have several adverse effects. These include:

$>$ unnecessary treatment and investigation

$>$ missing or delayed surgery

$>$ unnecessary isolation and contact tracing with subsequent negative impact on workforce and resources

$>$ a risk of subsequent increased exposure if the individual changes their behaviour as a result of believing that they have been infected

> the individual being placed with other inpatients with COVID-19 and consequently exposed to the virus.

A major risk of a false positive result occurs when the individual is cohorted with other patients suffering from COVID-19 and is consequently exposed to the virus (Box 1).

Cohorting patients with positive results is unavoidable during periods of very high prevalence in most settings. Nonetheless, infection control measures to reduce the risk of spread from one patient to another should remain in place even in positive cohort 
Box 1. A case of an individual with a false positive COVID-19 result during a period of increased prevalence

Recently, the prevalence of COVID-19 has increased in our locality. Cohorting of suspect and proven patients with a positive result is now necessary. We managed a female patient who tested positive at low level in a single gene (RDRP). She was therefore cohorted into a bay of patients with positive SARS-CoV-2 results. Her result was subsequently viewed by the infectious diseases team who concluded that her result in combination with her clinical history was not compatible with COVID-19. She was therefore re-swabbed and moved out of the positive bay. Two subsequent tests were negative, and the patient did not develop symptoms compatible with COVID-19. Although the patient was moved out of the bay, this did not happen before some exposure may have occurred. Fortunately, however, this individual did not develop COVID-19 but the case serves to highlight the risks of false positive results in this setting.

areas, with recognition that some patients may have been placed on the unit as a consequence of a false positive test. These can include safe distancing of beds, use of face coverings when possible, regular cleaning of the area and encouraging patients not to mix with one another.

\section{Challenges of false positive and false negative results}

COVID-19 provides a unique challenge because the prevalence of the disease is changing in real time and in line with prevention measures (principally lockdowns). This moving prevalence impacts on testing strategies and the interpretation of results. It also enables clinicians to witness the effects of prevalence and interpretation of results based on PPV and NPV in real time.

The possibility of both false positive and false negative results must be considered at all times. In high-prevalence settings false negative results will be proportionally more of an issue. Conversely in low prevalence settings false positive results are proportionally greater. However, in both settings, both types of result are possible, are associated with potential adverse outcome and as such must be taken into account.

\section{Causes of false positive results and recommended measures to minimise them}

There are many potential causes of a false positive result, including the following:

> Mislabelling at the point of collection and at the point of processing. ${ }^{2}$ This can be guarded against by robust processes such as rigorous sampling and laboratory protocols.

> Contamination during sampling and processing. ${ }^{2}$ Having skilled and well-trained personnel is crucial to keeping this type of error rate low. Additionally, having stricter standards imposed in laboratory processes and testing including external quality assessment schemes and internal quality systems may help reduce the risk of this happening to a minimum.

> Low-level reactions in the PCR process, which may be generated for several reasons. ${ }^{2}$ Results with a single positive gene at low level $(\mathrm{Ct}>35)$ should therefore be treated with caution. Clear evidence-based guidelines on interpretation of low-level positive results should be developed for clinicians to become familiar with. Also, laboratories should report the details of the result to facilitate better interpretation at the bedside.

\section{Conclusion}

False positive results have the potential to cause harm in both high- and low-prevalence settings. Prevalence and the risk of harm needs to be considered when deciding on testing strategies. We believe that testing strategies need to be more agile and decisions on screening of various populations should be flexible and respond to the changing prevalence in the community or setting that is being investigated. Routine large-scale screening has the potential to cause the most harm in this respect and this risk needs to be balanced against the benefit that it will afford in any given setting. Large-volume screening at a time of low prevalence has the potential to do more harm than good and some largescale screening strategies should be temporarily suspended when prevalence is very low. These strategies are likely to be of greater benefit in interrupting transmission during periods of high prevalence and we propose that they are re-instated when the prevalence in the community or particular settings warrant such an approach.

Careful interpretation of laboratory results is also required at all times, particularly in the context of screening asymptomatic individuals and cohorting positive patients. Low-level positive results in a single gene need to be interpreted with caution based on the clinical context. The $\mathrm{Ct}$ value can also provide useful information when assessing results and Clinicians need to become familiar with the interpretation of these results. Results should also be conveyed detailing the number of genes positive and the $\mathrm{Ct}$ value - not simply in a binary fashion (positive or negative).

\section{Acknowledgements}

We would like to acknowledge all the virology and microbiology laboratory staff who performed these tests.

\section{References}

1 Watson J, Whiting PF, Brush JE. Interpreting a Covid-19 test result. BMJ 2020;369:m1808.

2 Mayers C, Baker K. Impact of false-positives and false-negatives in the UK's COVID-19 RT-PCR testing programme. Government Office for Science, 2020. Available from www.gov.uk/government/ publications/gos-impact-of-false-positives-and-negatives-3-june2020 [Accessed 20 September 2020].

3 Parikh R, Mathai A, Parikh S, Chandra Sekhar G, Thomas R. Understanding and using sensitivity, specificity, and predictive values. Indian J Ophthal 2008;56:45.

4 Holborow A, Asad H, Porter L et al. The clinical sensitivity of a single SARS-CoV-2 upper respiratory tract RT-PCR test for diagnosing COVID-19 using convalescent antibody as a comparator. Clin Med 2020;20:e209-11.

5 Tenny S, Hoffman MR. Prevalence. StatPearls, 2020. Available from www.ncbi.nlm.nih.gov/books/NBK430867/ [Accessed 20 September 2020].

Address for correspondence: Dr Brendan Healy, Public Health Wales Microbiology (Cardiff and Swansea), University Hospital of Wales, Heath Park, Cardiff CF14 4XW, UK. Email: brendan.healy@wales.nhs.uk 